\title{
Stress and the progression of the developmental
}

\section{hypothesis of schizophrenia}

\author{
DAVID COTTER and CARMINE M. PARIANTE
}

The neurodevelopmental hypothesis of schizophrenia proposes that an early insult to the developing brain could result in brain changes that are 'fixed', 'non-progressive' and lie 'dormant' until made manifest in adolescence or early adulthood. This hypothesis was promoted most recently in the 1980s (Lewis \& Murray, 1987; Weinberger, 1987), but similar models were proposed by earlier researchers (Clouston, 1891; Southard, 1915; Pasamanick et al, 1956). The success of this theory has been in its capacity to explain many of the known features of schizophrenia. Thus, the season-of-birth effect, the reported excess of obstetric complications, minor physical anomalies, dermatoglyphic abnormalities and childhood developmental impairments may all be successfully and usefully explained in relation to the influence of environmental and epigenetic factors on normal foetal brain development. Crucial supportive evidence for the theory was given by studies showing that classical degenerative brain changes were largely absent, by the presence of microscopic brain changes indicative of abnormal brain development, and by the absence of clear evidence for progressive ventricular dilation among people with schizophrenia.

However, these latter findings are no longer secure. For example, the absence of excess cortical gliosis is no longer accepted as indicating a lack of perinatal or postnatal cerebral inflammation, and firm cytoarchitectural evidence of abnormal brain development has yet to be consistently presented (Harrison, 1999). Furthermore, some neuroimaging studies have shown evidence for progressive brain volume reductions over time (Mathalon et al, 2001) and others have shown that hippocampal volume reductions occur during the phase of a first psychotic episode (Lawrie et al, 2000; Pantellis et al, 2000). Reversibility of superior temporal gyrus volume reductions has also been described during the first episode of psychosis (Keshavan et al,
1998), and progressive cortical reductions are observed in people with early-onset schizophrenia (Thompson et al, 2001). Thus, evidence is accumulating to support the view that plastic changes in macroscopic cytoarchitecture may occur with illness.

The microscopic basis of these cortical volume reductions is not known, but clues are provided by recent neuropathological studies which show reductions in cortical neuronal size, dendritic complexity and synaptic proteins in schizophrenia (Harrison, 1999). Smaller neurons with fewer and less elaborate branches would result in a diminution in the amount of neuropil, more compacted cells, and thus increased neuronal density. It is certainly feasible to suggest that these alterations underlie the regional cortical volume reductions observed in schizophrenia. The current challenge, however, is to understand the mechanism underlying these changes. The term 'atrophic' is preferred to 'degenerative' because evidence of cell death and of the gliotic response so characteristic of degenerative diseases is absent. However, regardless of terminology, the findings necessitate a re-evaluation of the neurodevelopmental hypothesis of schizophrenia as described by Lewis \& Murray (1987).

\section{EVIDENCE FOR A COMMON NEUROPATHOLOGY IN MAJOR DEPRESSION, BIPOLAR DISORDER AND SCHIZOPHRENIA}

The microscopic neuropathological changes in schizophrenia may be either a vulnerability factor, or an intrinsic component or consequence of the illness. Neuropathological studies cannot tell which interpretation is correct. Some histological changes, such as those of reduced neuronal density in bipolar disorder but not in schizophrenia (Rajkowska et al, 2001) and alterations in hippocampal kainate receptor function in schizophrenia but not in bipolar disorder (Benes et al, 2001) argue for disease-specific processes. However, similarities in the neuropathology of schizophrenia, major depressive disorder and bipolar disorder suggest that a common process of change might be involved. These findings are not discordant, for they argue that the observed pathology in these disorders might be the product of both disease-specific and non-specific processes.

What pathological changes are shared? First, macroscopic neuroanatomical investigations of brain changes in schizophrenia, bipolar disorder and major depressive disorder show differences that are generally quantitative rather than qualitative. For example, ventricular dilation and reduced hippocampal and frontal brain volumes are seen in schizophrenia, but they are also present to a lesser degree in major depressive disorder and bipolar disorder (McCarley et al, 1999). The single main departure from this pattern is that the volume of the amygdala may be specifically enlarged in bipolar disorder (Altshuler et al, 1998), possibly because of drug treatment, although this is not yet resolved. Second, at the microscopic level, reductions in dendritic spine density (Rosoklija et al, 2000), neuronal size (Rajkowska et al, 1999; Cotter et al, 2001a, 2002) and synaptic proteins (Eastwood \& Harrison, 2001) have been described in both mood disorders and schizophrenia (Harrison, 1999). Finally, it has now become apparent that glial cell deficit may also be a feature of major depressive disorder, bipolar disorder and schizophrenia (Rajkowska et al, 1999; Cotter et al, 2001a,b, 2002), depending possibly on the coexistence of affective symptoms and on which region of the brain is investigated. Although the latter finding's functional and aetiological significance is not yet clear, it further demonstrates a similar brain pathology in these disorders and may provide an important clue to identifying aspects of the underlying disease processes.

The similarity of these patterns of changes in cortical cellular architecture in schizophrenia and mood disorders suggests that a common biological mechanism may underlie aspects of these psychiatric diseases. What aspects of illness common to major depressive disorder, bipolar disorder and schizophrenia could cause changes in keeping with the known cellular changes described above? We do not propose that 
this common process is necessarily primary to the pathophysiology of these disorders rather, that a single biological mechanism might participate in an important way in the development of the observed neuropathological (micro- and macroscopic) abnormalities. Glucocorticoid-related neurotoxicity is a candidate that needs to be considered.

\section{ROLE OF \\ GLUCOCORTICOIDS}

There is substantial evidence that hyperactivity of the hypothalamic-pituitaryadrenal (HPA) axis is involved in the pathogenesis of mood disorder (Pariante $\&$ Miller, 2001). Impaired function of the glucocorticoid receptor and subsequent altered feedback inhibition by endogenous glucocorticoids probably represents the mechanism by which the HPA axis is activated in depression. In contrast, until recently there was no firm evidence that HPA hyperactivity is part of the pathogenesis of schizophrenia. The rate of non-suppression to the dexamethasone suppression test in schizophrenia varies from $0 \%$ to $70 \%$, with a mean rate of approximately $20 \%$ (Sharma et al, 1988), which is much lower than that described in depression (Evans et al, 1983) but still higher than in the normal population (Sharma et al, 1988). The range appears to reflect the type of patient, the activity of the patient, the presence of associated symptoms of depression and the effect of hospitalisation. Indeed, in schizophrenia there is evidence that patients who are clinically stable and receiving treatment tend to have a normal HPA axis (Tandon et al, 1991; Ismail et al, 1998), but patients who are drug-free or in the acute phase of the illness have an activated HPA axis (Holsboer-Trachsler et al, 1987; Tandon et al, 1991). This suggests, crucially, that the inconsistencies of the HPA axis changes observed in schizophrenia may be because fewer studies have assessed patients during the acute phase of the schizophrenic illness, which is the very period most likely to be associated with a stressrelated elevation of glucocorticoids. Moreover, reduced glucocorticoid receptor gene expression has been described in the frontal cortex in schizophrenia and major depressive disorder (Webster et al, 2000), providing some evidence that HPA axis abnormalities may indeed be present in both these disorders, albeit mediated by different mechanisms.
Several other lines of investigation support the view that glucocorticoid-related neurotoxicity may be implicated in depression and schizophrenia. First, investigations in vitro have shown that high levels of glucocorticoid hormones result in reduced neuronal volume and dendritic arborisation (Sapolsky, 2000), and these changes have been observed in both disorders. Second, elevated plasma glucocorticoid levels are associated with hippocampal volume reductions in major depressive disorder, post-traumatic stress disorder, Cushing's disease and normal ageing, and such reductions have been observed in the phase of a first psychotic episode (Lawrie et al, 2000; Pantellis et al, 2000). Third, the functional effect of glucocorticoids on reducing hippocampal glial cell activation and proliferation (Crossin et al, 1997) mirrors the glial deficit observed in major depressive disorder, bipolar disorder and possibly schizophrenia. Consequently the glial deficit found in these disorders may also relate to glucocorticoid-related effects.

At present one can only speculate whether this putative link between glucocorticoid hormones and macroscopic brain changes in schizophrenia is exerted mainly through permanent damage of the brain cells or through reversible changes in the neuropil structure. In animals, stressinduced changes in dendritic arborisation are reversible after the stressor is eliminated, unless the stress is severe and protracted over weeks or months (Sapolsky, 1996). Moreover, the hippocampal volume reduction in people with Cushing's disease is also reversible after normalisation of cortisol levels (Sapolsky, 2000). Evidence in psychiatric disorders is less clear and the mechanisms may be different in different phases of the disorders. For example, the temporal gyrus volume reductions described during the first episode of psychosis seem to be reversible (Keshavan et al, 1998), but the hippocampal reductions described in patients with a long history of major depressive disorder are present even in stable patients with normal cortisol levels, and are correlated with the length of illness (Sapolsky, 2000). Reassuringly, a recent study has found minimal or no evidence of apoptosis in the post-mortem analysis of the hippocampus from patients with major depression (Lucassen et al, 2001), suggesting that the main alterations may involve potentially reversible changes to neuropil, glia and dendrites.
Despite this evidence supporting the view that interactions between glucocorticoid hormones and the brain might be abnormal in major depressive disorder and schizophrenia, there remain some unanswered questions. First, it is not at all clear whether the mechanism of neurotoxicity in vivo is mediated through elevated or lowered levels of cerebral glucocorticoids, for very low levels also have neurotoxic effects (Sapolsky, 2000). Second, reduced glucocorticoid receptor function is observed in individuals with major depression (Pariante \& Miller, 2001) or undergoing severe psychological stress (Bauer et al, 2000), suggesting that elevated plasma cortisol levels could represent a compensatory strategy. Third, studies indicate that levels of cortisol in the human brain are regulated by efflux systems at the blood-brain barrier (De Kloet et al, 1998), and that both the glucocorticoid receptor and the cortisol efflux systems may be influenced by psychotropic drugs (Pariante \& Miller, 2001; Pariante et al, 2001). This indicates that peripheral cortisol levels may not necessarily dictate cerebral levels, as is often assumed in studies. Finally, adjuvant antidepressant effects have been demonstrated not only with compounds that stimulate glucocorticoid receptor function (e.g. dexamethasone, prednisolone) but also with compounds that inhibit its function (e.g. RU486, ketoconazole). Therefore, whether patients with major depression or schizophrenia have elevated or lowered activation of the glucocorticoid receptor within the brain is yet to be fully elucidated.

Further development of this hypothesis will require research into HPA function in these disorders, such as post-mortem brain investigations into levels of corticotrophinreleasing hormone in the paraventricular nucleus of the thalamus in schizophrenia and bipolar disorder, and in vivo study of the relationship between glucocorticoid receptor function, cortisol levels and brain changes in patients with depression and schizophrenia at various stages of these illnesses.

\section{CONCLUSION}

Evidence is accumulating that brain changes occur during and possibly after the period of the first acute psychosis changes that are not purely developmental in the traditional sense. Furthermore, these 
alterations are not specific to schizophrenia, in terms of either macroscopic or microscopic brain structure, for they are also present to a generally milder degree in people with mood disorder, and are in keeping with glucocorticoid-related brain changes. Although it is possible even likely - that these brain changes are secondary to stress-related changes in glucocorticoid hormones rather than primary pathogenetic pathways, they may none the less have crucial clinical effects through diminishing neuronal and cortical function. This is because these brain changes may complicate recovery from the primary illness. In the future, it may be possible to reverse these changes by therapies that protect against glucocorticoid-related neurotoxicity or promote neuroprotective cell signalling pathways. In the meantime, however, the developmental theory of schizophrenia as presented by Lewis \& Murray (1987) and Weinberger (1987) is challenged, for alone it is insufficient to explain the unfolding neuroanatomy of the disorder, which now seems likely to involve both early developmental and later atrophic processes. Targeting these later processes may offer crucial therapeutic opportunities.

\section{DECLAR ATION OF INTEREST}

D.C. is supported by a Wellcome Trust University Award and by the Stanley Foundation.

\section{REFERENCES}

Altshuler, L. L., Bartzokis, G., Grieder, T., et al (1998) Amygdala enlargement in bipolar disorder and hippocampal reduction in schizophrenia: an MRI study demonstrating neuroanatomic specificity. Archives of General Psychiatry, 55, 663-664.

Bauer, M. E., Vedhara, K., Perks, P., et al (2000) Chronic stress in caregivers of dementia patients is associated with reduced lymphocyte sensitivity to glucocorticoids. Journal of Neuroimmunology, 103, 84-92.

Benes, F. M., Todtenkopf, M. S. \& Kostoulakos, P. (200I) GluR5,6,7 subunit immunoreactivity on apical pyramidal cell dendrites in hippocampus of

schizophrenics and manic depressives. Hippocampus, II 482-491.

Clouston, T. S. (189I) The Neuroses of Development. Edinburgh: Oliver \& Boyd.

Cotter, D., Mackay, D., Landau, S., et al (200la) Glial cell loss and reduced neuronal size in the anterior cingulate cortex in major depressive disorder. Archives of General Psychiatry, 58, 545-553.

, Pariante, C. M. \& Everall, I. (200Ib) Glial cell abnormalities in major psychiatric disorders: the evidence and implications. Brain Research Bulletin, 55, 585-595.

DAVID COTTER, MRCPsych, CARMINE M. PARIANTE, MRCPsych, Department of Psychological Medicine, Institute of Psychiatry, London, UK

Correspondence: Dr David R. Cotter, Department of Psychological Medicine, Institute of Psychiatry, DeCrespigny Park, London SE5 8AF, UK. Tel: 020 7919 3566/3285; fax: 0207919 2199; e-mail: david.cotter@iop.kcl.ac.uk

(First received II September 200I, final revision 18 April 2002, accepted 23 April 2002)

_, Mackay, D., Chana, G., et al (2002) Reduced neuronal size and glial cell density in area 9 of the dorsolateral prefrontal cortex in subjects with major depressive disorder. Cerebral Cortex, I2, 386-394.

Crossin, K. L., Tai, M. H., Krushel, L. A., et al (1997) Glucocorticoid receptor pathways are involved in the inhibition of astrocyte proliferation. Proceedings of the National Academy of Sciences of the USA, 94, 2687-2692.

De Kloet, E. R., Vreugdenhil, E., Oitzl, M. S., et al (1998) Brain corticosteroid receptor balance in health and disease. Endocrine Reviews, 19, 269-30I.

Eastwood, S. L. \& Harrison, P. J. (200I) Synaptic pathology in the anterior cingulate cortex in schizophrenia and mood disorders. A review and a western blot study of synaptophysin, GAP-43, and the complexins. Brain Research Bulletin, 55, 569-578.

Evans, D. L., Burnett, G. B. \& Nemeroff, C. B. (1983) The dexamethasone suppression test in the clinical setting. American Journal of Psychiatry, 140, 586-589.

Harrison, P. J. (1999) The neuropathology of schizophrenia: a critical review of the data and their interpretation. Brain, 122, 593-624.

Holsboer-Trachsler, E., Buol, C., Wiedemann, K., et al (1987) Dexamethasone suppression test in severe schizophrenic illness: effects of plasma dexamethasone and caffeine levels. Acta Psychiatrica Scandinavica, 75 , 608-613.

Ismail, K., Murray, R. M., Wheeler, M. J., et al (1998) The dexamethasone suppression test in schizophrenia. Psychological Medicine, 28, 3II-3I7.

Keshavan, M. S., Haas, G. L., Kahn, C. E., et al (1998) Superior temporal gyrus and the course of early schizophrenia: progressive, static or reversible? Journal of Psychiatric Research, 32, 161-167.

Lawrie, S. M., Whalley, H., Byrne, M., et al (2000) Brain structure change and psychopathology in subjects at high risk of schizophrenia. Schizophrenia Research, 4I,

Lewis, S.W. \& Murray, R. M. (1987) Is schizophrenia a neurodevelopmental disorder? BMJ, 295, 68I-682.

Lucassen, P. J., Muller, M. B., Holsboer, F., et al (200I) Hippocampal apoptosis in major depression is a minor event and absent from subareas at risk for glucocorticoid overexposure. American Journal of Pathology, I58, 453-468.

Mathalon, D. H., Sullivan, E.V., Lim, K. O., et al (200I) Progressive brain volume changes and the clinica course of schizophrenia in men: a longitudinal magnetic resonance imaging study. Archives of General Psychiatry, 58, $148-157$

McCarley, R.W., Wible, C. G., Frumin, M., et al (1999) $\mathrm{MRI}$ anatomy of schizophrenia. Biological Psychiatry, $\mathbf{4 5}$, 1099-III9.

Pantellis, C., Velakoulis, D., Suckling, J., et al (2000) Left medial temporal lobe volume reduction occurs during the transition from high risk to first episode psychosis. Schizophrenia Research, 4I, 35.
Pariante, C. M. \& Miller, A. H. (200I) Glucocorticoid receptors in major depression: relevance to pathophysiology and treatment. Biological Psychiatry, 49, 391-404.

_ , Makoff, A., Lovestone, S., et al (200I) Antidepressants enhance glucocorticoid receptor function in vitro by modulating the membrane steroid transporter. British Journal of Pharmacology, 134 1335-1343.

Pasamanick, B., Rodgers, M. E. \& Lilienfield, A. M. (1956) Pregnancy experience and behaviour disorder in children. American Journal of Psychiatry, I12, 613-618.

Rajkowska, G., Miguel-Hidalgo, J. J. \& Wei, J. (1999) Morphometric evidence for neuronal and glial prefrontal cell pathology in major depression. Biological Psychiatry, 45, 1085-1098.

_, Halaris, A. \& Selemon, L. D. (200I) Reductions in neuronal and glial density characterize the dorsolateral prefrontal cortex in bipolar disorder. Biological Psychiatry, 49, 741-752.

Rosoklija, G., Toomayan, G., Ellis, S. P., et al (2000) Structural abnormalities of subicular dendrites in subjects with schizophrenia and mood disorders. Archives of General Psychiatry, 57, 349-356.

Sapolsky, R. (1996) Stress, glucocorticoids, and the damage to the nervous system: the current state of confusion. Stress, I, I-II.

(2000) The possibility of neurotoxicity in the hippocampus in major depression: a primer on neuron death. Biological Psychiatry, 48, 755-765.

Sharma, R. P., Pandey, G. N., Janicak, P. G., et al (1988) The effect of diagnosis and age on the DST: a metaanalytic approach. Biological Psychiatry, 24, 555-568.

Southard, E. E. (1915) On the topographical distribution of cortex lesions and anomalies in dementia praecox with some account of their functional significance. American Journal of Insanity, 7I, 603-67I.

Tandon, R., Mazzara, C., DeQuardo, J., et al (1991) Dexamethasone suppression test in schizophrenia: relationship to symptomatology, ventricular enlargement, and outcome. Biological Psychiatry, 29, 953-964.

Thompson, P. M., Vidal, C., Giedd, I. N., et al (200I) Mapping adolescent brain change reveals dynamic wave of accelerated gray matter loss in very early-onset schizophrenia. Proceedings of the National Academy of Sciences of the USA, 98, I1650-11655.

Webster, M. J., O'Grady, J., Orthmann, C., et al (2000) Decreased glucocorticoid receptor mRNA levels in individuals with depression, bipolar disorder and schizophrenia. Schizophrenia Research, 4I, III.

Weinberger, D. R. (1987) Implications of normal brain development for the pathogenesis of schizophrenia. Archives of General Psychiatry, 44, 660-669. 